\title{
Prognostic clinical and radiological parameters for immediate facial nerve function following vestibular schwannoma surgery
}

\author{
Venelin Miloslavov Gerganov • \\ Petra Margarete Klinge • Mohsen Nouri • \\ Lennart Stieglitz $\cdot$ Madjid Samii $\cdot$ Amir Samii
}

Received: 8 May 2008/Accepted: 12 December 2008/Published online: 1 April 2009

(C) Springer-Verlag 2009

\begin{abstract}
Objective The paper aims to define the parameters available before surgery which could predict immediate facial nerve function after excision of a vestibular schwannoma (VS).

Methods Ninety-nine patients with VS operated consecutively by a single surgeon using an identical surgical technique have been evaluated retrospectively. Data were collected regarding patients' sex, age at onset of symptoms and at surgery, initial symptoms, neurological status at presentation, early post-operative neurological status and complications. The main radiological parameters included in the study were tumour extension pattern, diameters, shape, and volume, as well as extent of bony changes of the internal auditory canal.

Results As the tumour stage and volume increase, facial nerve function is worse after surgery $(p<0.001$ and $p<0.05$, respectively). Concomitantly, larger extra-meatal tumour diameters in three dimensions (sagittal, coronal and axial) led to worse function $(p<0.01)$. Anterior and/or caudal tumour extension ( $p=0.001$ and $p=0.004$, respectively) had more significant correlation than posterior and/or cranial extension ( $p=0.022$ and $p=0.353$, respectively). Polycyclic VS had the worst prognosis, followed by the tumours with
\end{abstract}

V. M. Gerganov $(\varangle) \cdot$ P. M. Klinge $\cdot$ L. Stieglitz $\cdot$ M. Samii $\cdot$ A. Samii

International Neuroscience Institute-Hannover,

Rudolf Pichlmayr Str. 4,

Hannover 30625, Germany

e-mail:vgerganov@gmail.com

M. Nouri

Tehran University of Medical Sciences,

Tehran, Iran oval shape. The extent of intra-meatal tumour growth does not correlate with immediate facial nerve outcome. The different angles, lengths and diameters of the internal auditory channel showed no significant correlation with facial nerve outcome. Patients with headache as an initial symptom and those with gait instability and/or preoperative poor facial nerve function had significantly worse immediate facial nerve outcome.

Conclusion Our data suggests that the analysis of the radiological and neurological patient data prior to surgery could give reliable clues regarding the immediate postoperative facial nerve function.

Keywords Vestibular schwannoma - Facial nerve · Surgical treatment $\cdot$ Prognostic parameter

The treatment of vestibular schwannomas (VS) has undergone significant evolution in the past several decades and the facial nerve is being preserved at increasingly higher rates. However $7-48 \%$ of the patients, following both microsurgery or radiosurgery, still experience temporary or permanent deterioration of facial nerve function $[10,13,17$, 22, 24, 34-36, 40]. Multiple studies have been performed in the quest of predictive parameters for facial nerve functional outcome and many criteria have been suggested, although their reliability remains controversial. The main shortcoming of most of the studies is the heterogeneity of the analysed series. They included patients operated over a long time frame by different surgeons. Furthermore, varying approaches and techniques of tumour removal and dissection of the facial nerve have been applied.

The aim of this study was to define pre-operatively assessable parameters that correlate with immediate facial 
nerve outcome following VS surgery via the retrosigmoid approach. Given the high rate of excellent and good facial nerve outcome at late follow-up that we have presented recently [35], an analysis of the prognostic parameters for late facial nerve function would not allow us to draw relevant conclusions. However, the current evaluation of early post-operative changes may reflect some of the aspects, including facial nerve vulnerability and/or increased nerve manipulation due to difficulty of facial nerve dissection. We performed an analysis of 99 patients with VS operated by a single neurosurgeon over a short period. The homogeneous characteristics of the group and the identical surgical technique applied allowed us to exclude some of the above mentioned treatment-related variables.

\section{Materials and methods}

\section{Clinical material}

A retrospective study of 99 consecutive patients with VS operated by a single neurosurgeon (MS) between May 2004 and December 2005 is presented. All patients underwent a standard pre-operative examination set and have been operated via the retrosigmoid approach. The technique of tumour removal was similar in all patients and has been described earlier in detail [32-35].

Data were collected regarding patient's gender, age at onset of symptoms and at surgery, initial symptoms, neurological status at presentation, intra-operative tumour characteristics, early post-operative neurological status, and complications. The facial nerve function was assessed 2 weeks after surgery according to the House-Brackmann scale [18]. The tumours classified according to the extension classification system developed in Hannover [33]: Class T1: intra-meatal tumour; Class T2: intra- and extra-meatal tumour; Class T3a: the tumour fills the cerebellopontine cistern; Class T3b: the tumour reaches the brainstem; Class T4a: the tumour compresses the brainstem; and Class T4b: the tumour severely dislocates the brainstem and compresses the fourth ventricle. The distribution of the patients according to their extension grade is shown in Table 1 . More than $46 \%$ of the VS in our series were in Class 4.

There were 51 females and 48 male patients. The average age at onset was $43.04 \pm 1.4$ years and the average age at surgery was $46.85 \pm 1.3$ years. The period between diagnosis and treatment was on average $37.11 \pm 4.4$ months. The most frequent initial sign was hearing deficit (68.7\%), followed by tinnitus $(25.3 \%)$, vertigo $(16.2 \%)$ and headache $(6.1 \%)$. At presentation, hearing deficit was noted in $81.4 \%$, tinnitus in $43.4 \%$, vertigo in $30.3 \%$, cerebellar signs in $22.2 \%$, and gait instability in $18.2 \%$.
Table 1 Distribution of the VS in accordance to the Hannover extension classification system

\begin{tabular}{lc}
\hline Tumour extension & Incidence (number) \\
\hline $\mathrm{T} 1$ & $9.1 \%(9)$ \\
$\mathrm{T} 2$ & $10.1 \%(10)$ \\
$\mathrm{T} 3 \mathrm{a}$ & $19.2 \%(19)$ \\
$\mathrm{T} 3 \mathrm{~b}$ & $15.2 \%(15)$ \\
$\mathrm{T} 4 \mathrm{a}$ & $33.3 \%(33)$ \\
$\mathrm{T} 4 \mathrm{~b}$ & $13.1 \%(13)$ \\
\hline
\end{tabular}

\section{Radiological analysis}

An imaging processing software (Image J 1.3b, NHI, USA) was used to measure the radiological parameters. Native and Gd-enhanced pre-operative MRI studies included T1and T2-weighted coronal, sagittal and axial sequences. The following aspects were measured: antero-posterior tumour diameter (parallel to the petrous ridge), medio-lateral diameter (perpendicular to the petrous ridge) and sagittal (vertical) diameter; cranial, caudal, anterior and posterior tumour extension in relation to the internal auditory canal (IAC); the width and length of the intra-meatal tumour portion; and the distance (the tumour-fundus distance) between the most lateral intra-canalicular tumour border to the fundus-Figs. 1 and 2. The shape of the extra-meatal tumour was defined based on MRI characteristics: oval,

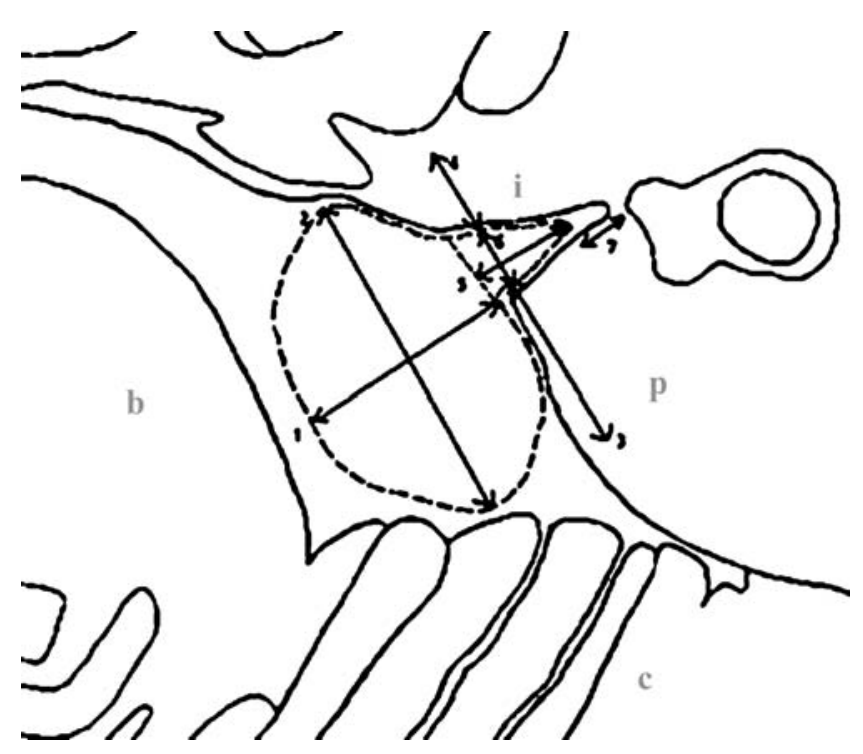

Fig. 1 Tumour measurements on axial MRI images. (1)-maximal medio-lateral diameter (perpendicular to the petrous ridge); (2) maximal antero-posterior diameter (parallel to the petrous ridge); (3)posterior tumour extension; (4) — anterior extension; (5) - intra-meatal portion width; (6) - intra-meatal portion length; (7)-distance between the most lateral intra-canalicular tumour border to the fundus (tumourfundus distance); $b=$ brain stem; $c=$ cerebellum; $p=$ petrous bone; $i=$ internal auditory canal 


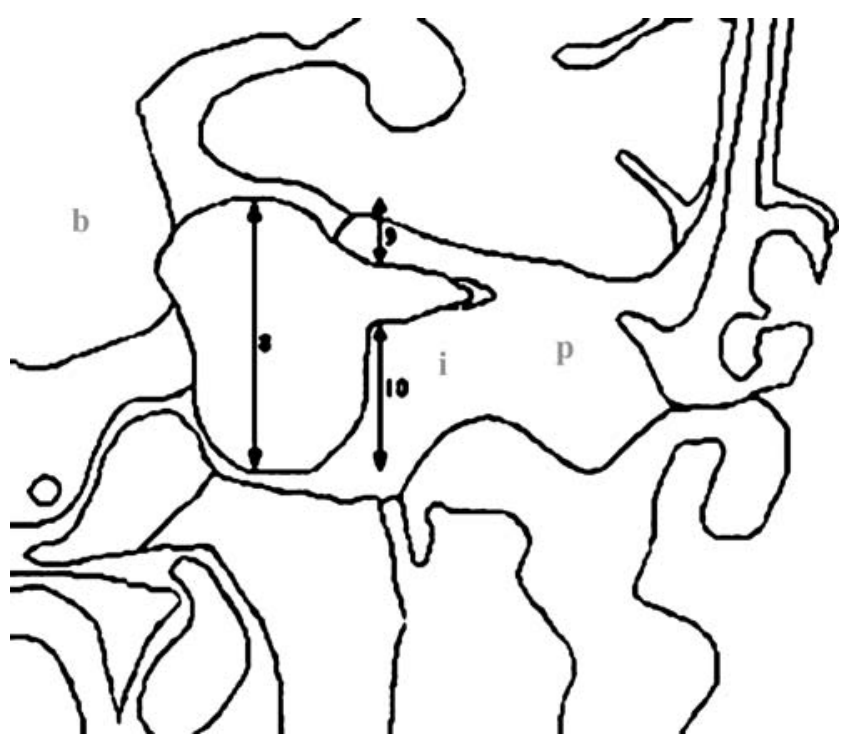

Fig. 2 Tumour measurements on coronal MRI images. (8) - maximal sagittal (vertical) diameter; (9) - cranial extension; (10)-caudal extension. $b=$ Brain stem; $p=$ petrous bone; $i=$ internal auditory canal

round and polycyclic. Oval VS were defined based on the axial shape on MR, where the shortest diameter had to be less than $90 \%$ of the longest diameter. If this relation was equal or greater than $90 \%$ the tumour was considered round. Cystic tumour changes were classified according to their size and localisation as microcysts or large cysts and superficial or deeply located cysts.

IAC changes were measured in pre-operative and postoperative bone window CT (slice thickness of $1 \mathrm{~mm}$ ). The measured parameters included: length of the anterior and posterior walls of the IAC; maximal diameter of the canal; its diameter measured at $90^{\circ}$ to the ICA axis; IAC lateral diameter, posterior semi-circular canal to IAC distance (Fig. 3), and IAC angle, defined on axial images.

Statistical analysis

Commercially available software (SPSS, version 13.0, Inc., Chicago, IL) was utilised for data analysis. In addition to parametric independent t-test and paired t-test, the nonparametric Kruskal-Wallis (KW) and Mann-Whitney U (MWU), Chi-square (CS) and Pearsons correlation tests were used. Significance was ascribed in case of error probability of $p<0.05$. All data are expressed as mean \pm standard error of mean.

\section{Results}

All the tumours have been removed totally. The anatomical integrity of the nerve was preserved in $98 \%(n=97)$ of the

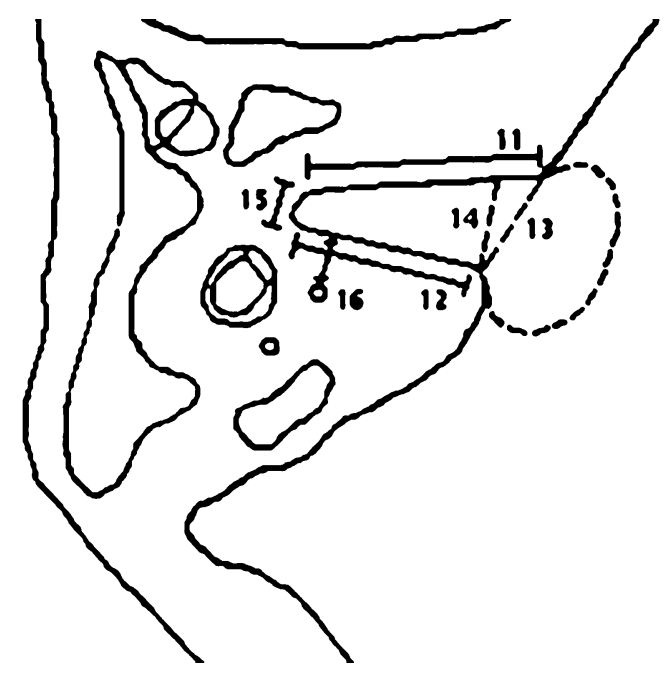

Fig. 3 Tumour-induced bony changes of the IAC. (11) - absolute length of the IAC anterior wall; (12) - absolute length of the IAC posterior wall; (13) - maximal IAC diameter; (14)-IAC diameter measured at 90 degrees to the IAC axis; (15)-lateral AC diameter; (16) - posterior semi-circular canal to auditory canal distance

patients (Table 2). In two patients intra-operative reconstruction of the nerve in the CPA was performed. Two weeks after surgery $78 \%$ of the patients had good or excellent facial nerve function, defined as HouseBrackmann Grades I-III. (Table 2).

The analysis of the clinical data showed that age at onset of initial symptoms and at surgery, symptoms duration, patient's gender, as well as pre-operative vertigo or tinnitus did not correlate with immediate post-operative facial function $(p>0.05)$. Patients with headache as an initial symptom had significantly worse facial nerve outcome (cs, mw). Similarly, gait instability at presentation correlated significantly with worse outcome (kw,mwu,cs). Trigeminal nerve dysfunction and lower cranial nerve deficits were associated with worse outcome, but the correlation was not found to be significant. Pre-operative hydrocephalus did not affect facial nerve outcome. Significant correlation existed between pre-operative and post-operative facial nerve function (cs,kw). No correlation was found between preoperative hearing level and post-operative facial nerve function, both with and without tumour stage exclusion and facial/hearing exclusion. On the other hand, significant correlation was found between post-operative hearing level and facial outcome, with and without tumour stage exclusion.

The results of the radiological analysis are presented in Table 3. Tumour stage was found to correlate positively with immediate facial nerve function $(p<0.001, \mathrm{cs}, \mathrm{kw})$. Tumour volume also correlated with facial outcome $(p<0.05$, Pearson: 0.278). Concomitantly, larger extrameatal tumour diameters in three dimensions (sagittal, 
Table 2 Pre-operative and early post-operative facial nerve function

\begin{tabular}{llccc}
\hline House-Brackmann Grade & Grade I & Grades II-III & Grades IV-V & Grade VI \\
\hline Pre-operative $(n=99)$ & $92(93 \%)$ & $4(4 \%)$ & $3(3 \%)$ & 0 \\
Post-operative $(n=99)$ & $52(53 \%)$ & $25(25 \%)$ & $19(19 \%)$ & $3(3 \%)$ \\
\hline
\end{tabular}

coronal and axial) led to worse function $(p<0.01)$. Tumour growth in all directions had significant correlation with immediate facial nerve outcome. Anterior tumour extension had more significant correlation than posterior extension (p:0.001, Pearson: 0.404 vs. p:0.022, Pearson: 0.282) and caudal extension had more significant correlation than cranial extension (p:0.004, Pearson: 0.353 vs. p:0.018, Pearson 0.291). The increase in tumour size and tumour volume correlated linearly with a gradual worsening facial function. However, regarding the tumour extension in the CPA, a breakthrough point after which the post-operative facial function became significantly worse existed. We found no significant difference between tumour stages up to stage T4a, i.e. MWU detected no significant difference between any two chosen stages less than T4a. However, tumour stages $\mathrm{T} 4 \mathrm{a}$ and $\mathrm{T} 4 \mathrm{~b}$ were associated with worse facial function compared with all other stages (MWU). Both intra-meatal tumour length and width showed no significant correlation to immediate outcome. The analysis showed that there are significant differences in facial nerve outcome among various tumour shapes (KW). Polycyclic VS had the worst prognosis, followed by the oval tumours $(p<0.05)$. Significant difference in facial nerve outcome was not found with round and intra-meatal tumours, as well as when different cystic tumour types were compared. Neither intra-meatal length nor intra-meatal width and tumour-fundus distance influenced the facial nerve function significantly.

The different angles and diameters of the IAC, as well as the posterior and anterior lengths of the walls showed no significant correlation with facial nerve outcome $(p>0.05$; Table 3).

\section{Discussion}

Facial nerve palsy, even if temporary, is one of the most troublesome impairments after VS treatment and a major factor determining the QOL of the patients [1, 7, 26, 38]. Different factors influencing the long-term facial nerve outcome after surgical treatment of VS have been discussed in the literature. Important parameters are the varying experience of the surgeons, the selected operative approach, and the extent of tumour removal [15, 27, 29, 37, 41]. In the current series, these surgery-related variables have been excluded. On the other hand, the conclusions from the
Table 3 Correlation between different radiological parameters and early post-operative facial nerve function

$H B$ House-Brackmann

\begin{tabular}{|c|c|c|c|c|}
\hline \multirow[t]{2}{*}{ Parameters } & \multicolumn{4}{|c|}{ Facial nerve function (House-Brackmann scale) } \\
\hline & HB I & HB II-III & HB IV-V & HB VI \\
\hline Medio-lateral diameter $(\mathrm{cm})$ & $1.53 \pm-0.15$ & $2.22 \pm 0.17$ & $2.04 \pm 0.18$ & $2.33 \pm 0.71$ \\
\hline Antero-posterior diameter $(\mathrm{cm})$ & $1.85 \pm 0.20$ & $2.71 \pm 0.26$ & $2.77 \pm 0.20$ & $2.48 \pm 0.12$ \\
\hline Sagittal diameter $(\mathrm{cm})$ & $1.80 \pm 0.18$ & $2.48 \pm 0.23$ & $2.44 \pm 0.16$ & $2.45 \pm 0.58$ \\
\hline Cranial extension $(\mathrm{cm})$ & $0.66 \pm 0.09$ & $1.03 \pm 0.13$ & $0.97 \pm 0.10$ & $0.94 \pm 0.09$ \\
\hline Caudal extension $(\mathrm{cm})$ & $0.56 \pm 0.06$ & $0.83 \pm 0.11$ & $0.86 \pm 0.08$ & $0.81 \pm 0.49$ \\
\hline Posterior extension (cm) & $0.70 \pm 0.10$ & $1.02 \pm 0.14$ & $1.03 \pm 0.11$ & $1.05 \pm 0.34$ \\
\hline Anterior extension $(\mathrm{cm})$ & $0.37 \pm 0.06$ & $0.69 \pm 0.11$ & $0.70 \pm 0.08$ & $0.83 \pm 0.20$ \\
\hline Intra-meatal length (cm) & $0.85 \pm-0.05$ & $0.98 \pm 0.08$ & $0.98 \pm 0.06$ & $0.88 \pm 0.05$ \\
\hline Intra-meatal width (cm) & $0.58 \pm 0.04$ & $0.70 \pm 0.05$ & $0.62 \pm 0.04$ & $0.65 \pm 0.02$ \\
\hline Tumour-fundus distance (cm) & $0.17 \pm 0.03$ & $0.19 \pm 0.04$ & $0.14 \pm 0.04$ & $0.19 \pm 0.02$ \\
\hline Tumour volume (ml) & $4.30 \pm 1.27$ & $8.36 \pm 1.92$ & $8.83 \pm 1.90$ & $7.50 \pm 3.19$ \\
\hline Maximal IAC diameter $(\mathrm{cm})$ & $1.10 \pm 0.03$ & $1.09 \pm 0.05$ & $1.13 \pm 0.05$ & $0.86 \pm 0.04$ \\
\hline $90^{\circ} \mathrm{IAC}$ diameter $(\mathrm{cm})$ & $0.84 \pm 0.03$ & $0.84 \pm 0.05$ & $0.87 \pm 0.07$ & $0.73 \pm 0.10$ \\
\hline three point angle (degrees) & $39.42 \pm 1.40$ & $39.61 \pm 2.18$ & $40.34 \pm 2.77$ & $34.18 \pm 4.5$ \\
\hline Lateral angle (degrees) & $53.27 \pm 1.57$ & $49.88 \pm 2.49$ & $49.71 \pm 2.35$ & $48.28 \pm 5.25$ \\
\hline Continued angle (degrees) & $29.38 \pm 1.60$ & $28.70 \pm 2.19$ & $28.66 \pm 2.34$ & $22.68 \pm 2.44$ \\
\hline Lateral IAC diameter $(\mathrm{cm})$ & $0.20 \pm 0.01$ & $0.18 \pm 0.01$ & $0.19 \pm 0.01$ & $0.24 \pm 0.01$ \\
\hline
\end{tabular}


study may be related to the retrosigmoid approach only. Most of the authors agree that the size of the tumour, its consistency and degree of adherence between the lesion and the nerve have a major influence on facial nerve outcome [2, 23, 27, 29, 31, 34].

Crucial among them is the size of the tumour $[9,20,21$, 37]. However, there is no general agreement on this point [21, 28]. Lalwani et al. [21] in a series of 121 patients with VS found no correlation between tumour size and facial nerve outcome. Moulin et al. [30] reported on 61 patients operated by the translabyrinthine approach and found no relationship between VS volume and the late post-operative facial motor function. However, the maximum tumour diameter measured by MRI was the best predictor of late facial nerve outcome. Our data shows that a significant correlation to immediate outcome exists with tumour stage, diameter and tumour volume. Concomitantly, larger extrameatal tumour diameters in three dimensions (sagittal, coronal and axial) led to worse facial function. However, anterior and/or caudal tumour extension had more significant correlation than posterior extension and/or cranial extension. The growth of VS usually results in a displacement of the facial nerve in the anterior and cranial direction $[25,36]$. Tumours with significant anterior extension could lead to more severe stretching and vulnerability of the nerve, which might be the explanation for the abovementioned correlations. The safest method for dissection of the facial nerve is to identify its lateral and medial portions, and then to dissect its medial portion by pulling the rest of the tumour capsule upward. A larger caudal tumour extension may aggravate the negative effect of the prolonged nerve stretching in one direction. The shape of the tumour also has a significant correlation with facial nerve outcome. VS with polycyclic shape had the worst prognosis, followed by oval tumours. Round tumours had the best prognosis. Although our study showed that the facial function depends on the size and extension of the extra-meatal tumour portion, the characteristics of the intrameatal portion, including tumour length, tumour width, tumour-fundus distance, as well as the bony changes of the ICA did not have significant relationship to immediate facial nerve outcome. Some authors reported that small VS/ IAC angle is associated with poorer post-operative facial function. The angulation of the facial nerve immediately next to the porus of the IAC might increase the risk of surgical trauma during dissection [8, 41] It could be assumed that an expanded IAC would decrease the facial nerve angulation and thereby lead to better outcome. However, our data showed that all parameters related to the shape and dimension of the IAC did not have any significant correlation with immediate facial nerve outcome. Similarly, Yamakami et al. [39] reported that the rate of good facial nerve function 1 year after surgery showed no significant correlation to the extent of IAC destruction and/or widening.

Cystic tumours have been reported to have an unfavourable prognosis $[5,25,30,41]$. In such lesions, the dissection of the facial nerve might be more difficult due to its marked distortion, unusual direction of displacement and tight adhesion to neural structures [3, 11, 27, 33]. Nevertheless, in the current study such a correlation was not found.

Previous studies have shown that some intra-operative electrophysiological parameters, related to EMG or auditory brainstem responses, could be used as predictors of facial nerve outcome $[4,8,12,14,16,19,21]$. Grayeli et al. [14] reported that facial function early after surgery was related to the nerve stimulation threshold at the brainstem and in the nerve-tumour adhesion zone. The intraoperative stimulation threshold correlates with the long-term functional outcome. Tumour-related predictive parameters to consider are presence of oedema and inflammation at light microscopic observation of the tumour specimen [6] and prior RT [27].

The analysis of patient's characteristics and clinical features, such as age, gender, symptom duration, and preoperative facial function, performed by Couloigner et al. [6], showed no correlation to post-operative facial nerve function. Some authors reported that increasing age was associated with poor facial outcomes [15]. A decision in favour of radiosurgery for older patients would be logical. Several other studies, however, did not prove that age has a negative predictive value $[6,21]$. The presented analysis showed that age at initial symptom and surgery, does not correlate with immediate post-operative facial function. Symptom duration before surgery and patient's gender did also not correlate with outcome. The only neurological symptoms at presentation that correlated significantly with worse immediate facial nerve function were gait instability and pre-operative facial palsy $(p<0.05)$. Trigeminal nerve dysfunction and lower cranial nerve deficit were associated with worse outcome, but the correlation was not found to be significant.

\section{Conclusion}

This study shows that there are specific radiological and neurological parameters that correlate significantly with immediate facial nerve outcome. Tumour shape, extrameatal tumour size, extension, and direction of growth are the most closely associated factors of post-operative facial nerve function. Intra-meatal growth pattern and IAC characteristics do not correlate with post-operative facial nerve function. Pre-operative gait instability and headache as the initial symptom also correlate positively. 


\section{References}

1. Anderson DE, Leonetti J, Wind JJ, Cribari D, Fahey K (2005) Resection of large vestibular schwannomas: facial nerve preservation in the context of surgical approach and patient-assessed outcome. J Neurosurg 102:643-649

2. Arriaga MA, Luxford WM, Atkins JS, Kwarlter JA (1993) Predicting long-term facial nerve outcome after acoustic neuroma surgery. Otolaryngol Head Neck Surg 108:220-224

3. Benech F, Perez R, Fontanella MM, Morra B, Albera R, Ducati A (2005) Cystic versus solid vestibular schwannomas: a series of 80 grade III-IV patients. Neurosurg Rev 28:209-213

4. Bozorg Grayeli A, Kalamarides M, Fraysse B, Deguine O, Favre G, Martin C, Mom T, Sterkers O (2005) Comparison between intra-operative observations and electromyographic monitoring data for facial nerve outcome after vestibular schwannoma surgery. Acta Otolaryngol 125:1069-1074

5. Charabi S, Tos M, Borgesen SE, Thomsen J (1994) Cystic acoustic neuromas: results of trans-labyrinthine surgery. Arch Otolaryngol Head Neck Surg 120:1333-1338

6. Couloigner V, Gervaz E, Kalamarides M, Ferrary E, Rey A, Sterkers O, Henin D (2003) Clinical and histologic parameters correlated with facial nerve function after vestibular schwannoma surgery. Skull Base 13:13-19

7. Cross T, Sheard CE, Garrud P, Nikolopoulos TP, O'Donoghue GM (2000) Impact of facial paralysis on patients with acoustic neuroma. Laryngoscope 110:1539-1542

8. Deguine O, Maillard A, Bonafe A, el Adouli H, Tremoulet M, Fraysse B (1998) Pre-operative and per-operative factors conditioning long-term facial nerve function in vestibular schwannoma surgery through trans-labyrinthine approach. J Laryngol Otol 112:441-445

9. Fenton JE, Chin RY, Shirazi A, Fagan PA (1999) Prediction of post-operative facial nerve function in acoustic neuroma surgery. Clin Otolaryngol 24:483-486

10. Flickinger JC, Kondziolka D, Niranjan A, Lunsford LD (2001) Results of acoustic neuroma radiosurgery: an analysis of 5 years' experience using current methods. J Neurosurg 94:1-6

11. Fundova P, Charabi S, Tos M, Thomsen J (2000) Cystic vestibular schwannoma: surgical outcome. J Laryngol Otol 114:935-939

12. Goldbrunner R, Schlake H, Milewski C, Tonn J, Helms J, Roosen K (2000) Quantitative parameters of intra-operative electromyography predict facial nerve outcomes for vestibular schwannoma surgery. Neurosurgery $46: 1140-1148$

13. Gormley WB, Sekhar LN, Wright DC, Kamerer D, Schessel D (1997) Acoustic neuroma: results of current surgical management. Neurosurgery 41:50-60

14. Grayeli AB, Guindi S, Kalamarides M, El Garem H, Smail M, Rey A, Sterkers O (2005) Four-channel electromyography of the facial nerve in vestibular schwannoma surgery: sensitivity and prognostic value for short-term facial function outcome. Otol Neurotol 26:114-120

15. Grey PL, Moffat DA, Palmer CR, Hardy DG, Baguley DM (1996) Factors which influence the facial nerve outcome in vestibular schwannoma surgery. Chir Otolaryngol 21:409-413

16. Hammerschlag PE, Cohen NL (1996) Intra-operative monitoring of facial nerve function in cerebellopontine angle surgery. Otolaryngol Head Neck Surg 103:681-684

17. Hasegawa T, Kida Y, Kobayashi T, Yoshimoto M, Mori Y, Yoshida J (2005) Long term outcomes in patients with vestibular schwannomas treated using gamma knife surgery: 10 year follow up. J Neurosurg 102:10-16

18. House JW, Brackmann DE (1985) Facial nerve grading system. Otolaryngol Head Neck Surg 93:146-147
19. Isaacson B, Kileny PR, El-Kashlan HK (2005) Prediction of longterm facial nerve outcomes with intra-operative nerve monitoring. Otol Neurotol 26:270-273

20. Kartush JM, Lundy LB (1992) Facial nerve outcome in acoustic neuroma surgery. Otolaryngol Clin North Am 3:623-647

21. Lalwani A, Butt F, Jackler R, Pitts L, Yingling C (1994) Facial nerve outcome after acoustic neuroma surgery: a study from the era of cranial nerve monitoring. Otolaryngol Head Neck Surg 111:561-570

22. Lanman TH, Brackmann DE, Hitselberger WE, Subin B (1999) Report of 190 consecutive cases of large acoustic tumours (vestibular schwannoma) removed via the trans-labyrinthine approach. J Neurosurg 90:617-623

23. Lee TKY, Lund WS, Adams CBT (1990) Factors infuencing the preservation of the facial nerve during acoustic surgery. $\mathrm{Br} \mathrm{J}$ Neurosurg 4:5-8

24. Lunsford LD, Niranjan A, Flickinger JC, Maitz A, Kondziolka D (2005) Radiosurgery of vestibular schwannomas: Summary of experience in 829 cases. J Neurosurg 102:195-199

25. Matthies C, Samii M, Krebs S (1997) Management of vestibular schwannomas (acoustic neuromas): radiological features in 202 cases - their value for diagnosis and their predictive importance. Neurosurgery 40:469-481

26. Magliulo G, Zardo F, Damico R, Varacalli S, Forino M (2000) Acoustic neuroma: post-operative quality of life. J Otolaryngol 29:344-347

27. McElveen JT Jr, Belmonte RG, Fukushima T, Bullard DE (2000) A review of facial nerve outcome in 100 consecutive cases of acoustic tumour surgery. Laryngoscope 110:1667-1672

28. Megerian CA, McKenna MJ, Ojemann RG (1996) Delayed facial paralysis after acoustic neuroma surgery: factors influencing recovery. Am J Otol 17:630-633

29. Moffat DA, Hardy DG, Grey PL, Baguley DM (1996) The operative learning curve and its effect on facial nerve outcome in vestibular schwannoma surgery. Am J Otol 17:643-647

30. Mulkens TH, Parizel PM, De Schepper AM, van de Heyning PH, Forton GE, Martin JJ, Delaporte C (1993) MRI of acoustic schwannoma: A retrospective study of 89 tumours. Rofo Fortschr Geb Rontgenstr Neuen Bildgeb Verfahr 158:362-367

31. Nutik SL (1994) Facial nerve outome after acoustic neuroma surgery. Surg Neurol 41:28-33

32. Samii M, Matthies C (1997) Management of 1,000 vestibular schwannomas (acoustic neuromas): clinical presentation. Neurosurgery 40:1-9

33. Samii M, Matthies C (1997) Management of 1000 vestibular schwannomas (acoustic neuromas): surgical management and results with an emphasis on complications and how to avoid them. Neurosurgery 40:11-21

34. Samii M, Matthies C (1997) Management of 1,000 vestibular schwannomas (acoustic neuromas): the facial nerve-preservation and restitution of function. Neurosurgery 40:684-695

35. Samii M, Gerganov V, Samii A (2006) Improved preservation of hearing and facial nerve function in vestibular schwannoma surgery via the retrosigmoid approach in a series of 200 patients. J Neurosurg 105:527-535

36. Sampath P, Holliday MJ, Brem H, Niparko JK, Long DM (1997) Facial nerve injury in acoustic neuroma (vestibular schwannoma) surgery: aetiology and prevention. J Neurosurg 87:60-66

37. Seol HJ, Kim CH, Park CK, Kim CH, Kim DG, Chung YS, Jung HW (2006) Optimal extent of resection in vestibular schwannoma surgery: relationship to recurrence and facial nerve preservation. Neurol Med Chir (Tokyo) 46:176-180

38. Strauss C, Romstock J, Fahlbusch R, Rampp S, Scheller C (2006) Preservation of facial nerve function after post-operative vasoactive treatment in vestibular schwannoma surgery. Neurosurgery 59:577-584 
39. Yamakami I, Uchino Y, Kobayashi E, Saeki N, Yamaura A (2002) Prognostic significance of changes in the internal acoustic meatus caused by vestibular schwannoma. Neurol Med Chir (Tokyo) 42:465-470

40. Yamakami I, Uchino Y, Kobayashi E, Yamaura A, Oka N (2004) Removal of large acoustic neurinomas (vestibular schwannomas) by the retrosigmoid approach with no mortality and minimal morbidity. J Neurol Neurosurg Psychiatry 75:453-458

41. Zaouche S, Ionescu E, Dubreuil C, Ferber-Viart C (2005) Pre- and intra-operative predictive factors of facial palsy in vestibular schwannoma surgery. Acta Otolaryngol 125:363-369

This is an interesting analysis of the prognostic factors for facial nerve outcome after vestibular schwannoma surgery. Preoperative clinical and radiological parameters were correlated with the outcome at two weeks postoperatively. Main negative factors were large size and direction of the tumor expansion, cystic structure and preexistig facial dysfunction.

Although these results are not all new the paper summarizes the important aspects well. In comparison to other articles the analysis comprises a homogeneous group of patients, almost 100 patients operated upon by one surgeon witnin one and a half year. The paper is well written and the pertinent literature is reflected.

H.J. Steiger

University of Düsseldorf

Facial Nerve Function following vestibular schwannoma surgery

The authors review a recent series of 99 patients with vestibular schwannomas who had tumor resection by Professor Samii via the retrosigmoid approach. They have specifically reviewed those preoperative clinical and imaging characteristics which could have been associated with facial nerve functional outcomes. They note that tumor size, especially larger extrameatal tumor volume,negatively impacted facial nerve outcome. Anterior extent of tumor was also negatively associated. Intrameatal tumor shape and size was not associated with outcome. Not surprisingly, gait instability and preoperative facial nerve dysfunction were associated with a poorer facial nerve outcome.Overall, these results are somewhat intuitive, however the strength of the submission was the uniformity of surgical factors, and experience of the surgical team which reduces these other variables relating to facial nerve outcome.

W.T. Couldwell

USA 